\title{
Experimental investigation of granular friction behaviors during reciprocating sliding
}

\author{
Xuejie ZHANG, Wei SUN, Wei WANG, Kun LIU* \\ Institute of Tribology, School of Mechanical Engineering, Hefei University of Technology, Hefei 230009, China \\ Received: 29 March 2020 / Revised: 31 October 2020 / Accepted: 07 January 2021 \\ (C) The author(s) 2021.
}

\begin{abstract}
Granular friction behaviors are crucial for understanding the ubiquitous packing and flow phenomena in nature and industrial production. In this study, a customized experimental apparatus that can simultaneously measure the time history of normal and tangential forces on the inside-shearing unit is employed to investigate the granular friction behaviors during a linear reciprocating sliding process. It is observed that the evolution behaviors of two normal forces distributed separately on the shearing unit can qualitatively reflect the effects of the force chain network. During the half-loop of the reciprocating sliding, the total normal force, which indicates the load-bearing capacity of the granular system, experiences the following typical stages: decreases abruptly and stabilizes momentarily, further decreases significantly to the minimum, gradually increases to the maximum, and then remains stable. These stages are associated closely with the relaxation, collapse, reconstruction, and stabilization of the force chain, respectively. Interestingly, the coefficient of friction (COF) can reach a stable value rapidly within the initial sliding stage and subsequently remain constant. The average COF within stable ranges decreases significantly with the external load $G$ in the power-function form, $G^{-0.5}$. Meanwhile, the COF increases slightly with the sliding velocity. Finally, a complete illustration of the dependences of the granular COF on the external load and sliding velocity is provided. Our study contributes to granular friction research by providing an innovative experimental approach for directly measuring the COF and implicitly correlating the evolution of the force chain network.
\end{abstract}

Keywords: granular friction; coefficient of friction; force chains; reciprocating sliding

\section{Introduction}

Granular friction is vital to the ubiquitous packing and flow phenomena in nature and industrial production, such as geographical faults [1, 2], powder mixing and densification [3], and granular flow lubrication $[4,5]$. The coefficient of friction (COF) is expressed as the tangential friction force divided by the total normal force and is typically used to describe the shear strength of a granular system [6-9]. Currently, granular friction research is associated with two experimental schemes, as follows: (1) the surface-sliding scheme, where the shearing unit is sliding against the surface of the granular system [10-15]; (2) the inside-sliding scheme, where the shearing unit is sliding inside the granular system [16-18].

Hitherto, the surface-sliding scheme has been employed extensively in granular friction studies owing to its simplicity. In addition, because the normal force applied to the shear surface is typically fixed in this scheme, the COF can be directly measured. For example, Nasuno et al. [10] measured the instantaneous friction force of granular matter

* Corresponding author: Kun LIU, E-mail: liukun@hfut.edu.cn 
under a low external load and discovered a functional relationship between the instantaneous friction force and instantaneous velocity. Géminard et al. [12] investigated the effect of the mixing ratio of dispersive particles on the friction force and COF in the stick-slip regime and demonstrated that the functional relationship between the COF and mixing ratio depended on the size ratio and shape of the two components. Siavoshi et al. [14] investigated the effect of granular layer thickness and shear surface roughness on the $\mathrm{COF}$ and discovered that the COF was related to the degree of particle restraint. Fall et al. [19] investigated the effect of liquid content on granular $\mathrm{COF}$ and reported that the COF was directly related to the shear modulus of the system. Azéma et al. [20] introduced a nanoindenter-based approach to investigate the effect of surface roughness on the sliding friction of micron-sized glass beads. These experiments indicated that the friction behavior of granular matter was affected by micro-scale (single particle) factors [21-23].

Moreover, the surface-sliding scheme has been adopted in studies that correlated granular rheology and granular friction. Based on numerous simulations and experiments, Jop et al. [8], and MiDi [13] discovered that, for rigid particles, the dependence of the friction coefficient on the rheological properties of granular matter can be determined by introducing a dimensionless inertial number $I=\dot{\gamma} d / \sqrt{P / \rho}$, where $\dot{\gamma}$ is the shear rate, $d$ the mean particle diameter, $P$ the normal stress, and $\rho$ the particle density. Based on the range of the inertial number $I$, the shear state of granular matter can be categorized into the following three regimes: quasi-static regime $\left(I<10^{-4}\right)$, inertial regime $\left(10^{-4}<I<10^{-1}\right)$, and collisional regime $\left(I>10^{-1}\right)$ [24]. In the inertial regime, granular matter flows like a liquid, and the particles experience enduring contact. Similarly, Iordanoff et al. [25] and Da Cruz et al. [26] also proposed that the effective COF could be described as a function of the inertial number I. Hatano [27] proposed a power friction law, in which the COF increased with the power of the inertial number such that $\mu(I)=\mu_{\mathrm{c}}+a_{\mu} I^{\alpha_{\mu}}$, where $\mu_{\mathrm{c}}$ denotes the friction coefficient for $\dot{\gamma} \rightarrow 0$, and $a_{\mu}$ is a constant. Additionally, Peyneau [18] discovered the exponent $\alpha_{\mu} \approx 1$ in numerous experiments using glass beads and sand as granular matter. Therefore, the effective COF was dependent on the rheological properties of granular matter.

Compared with the fixed normal load in the surface-sliding scheme, the normal force acting on the inside-sliding shearing unit is typically dynamic, owing to the evolution of the force chain network [28]. For granular matter, a force chain is a linear or quasilinear cluster of at least three contacting particles, where the compressive stress is concentrated $[29,30]$. Force chains can be regarded as stress transmission paths within the system that affect the load capacity significantly [31]. Subsequently, numerous force chains with different strengths randomly intersect with each other, constituting a force network that resides non-uniformly inside the granular assembly. The strength and topology of the force chains are sensitive to the applied load. Shearing motion can cause the continuous collapse and reconstruction of the force chain, resulting in dynamic changes in the force transmission paths and load capacity [32-35]. The inside-sliding scheme can be beneficial for revealing the effect of the force chain network on granular friction. However, the unknown dynamic normal force in the insidesliding scheme is difficult to measure, as is the COF. For example, Horváth et al. [16] measured only the static friction force when a glass rod was extracted from granular matter; however, they did not obtain the COF.

In this study, a customized experimental apparatus that overcomes the shortcomings of the current inside-sliding scheme was employed to investigate the friction characteristics of granular matter. Generally, forces between particles in a framework of granular matter include normal contact, and shear contact, capillary, cohesive, and van der Waals forces [33]. For our dry sliding process using millimeter-scale particles, only normal and shear contact forces were relevant $[13,36]$. The experimental apparatus can simultaneously measure the normal load and tangential friction forces acting on the shearing unit, thereby providing the time history of the 
dynamic COF during the entire reciprocating sliding process. The measured results can qualitatively reflect the evolution of the force chains at the mesoscopic scale. The effects of the external load and sliding velocity on granular friction behaviors are discussed herein.

\section{Experiment and method}

\subsection{Details of experimental apparatus}

In this study, granular friction experiments were performed using a customized linearly reciprocating friction apparatus. As shown in Fig. 1(a), the apparatus is primarily composed of a shearing box, a reciprocating sliding unit (sandwich structure), a linear motion stage, vertical position platforms, and other supporting parts. The shearing box $(80 \mathrm{~mm}$ $\times 100 \mathrm{~mm} \times 100 \mathrm{~mm}$ in length, width, and height, respectively) was constructed using $5 \mathrm{~mm}$-thick acrylic plates and used to contain the particles. In general, macroscopic experiments on granular matter primarily involve the use of millimeter-scale particles (ranging from $10^{-1}$ to $10^{1} \mathrm{~mm}$ ). Moreover, as confirmed in a few previous studies [37-39], the granular friction behavior is almost independent of the particle size in the stable slip regime. In practice, the number of particles should be sufficiently large in a specified container, and the particles should not slip out of the container from the side holes. Regarding these factors, approximately $6 \times 10^{4} \quad \mathrm{SiO}_{2}$ balls with a diameter ranging from 2.0 to $2.5 \mathrm{~mm}$ and a total mass of $1 \mathrm{~kg}$ were used as granular particles to fill the shearing box.

Two thin-film load sensors (sensors A and B) were sandwiched between the upper and lower splints, forming a core sandwich structure measuring $200 \mathrm{~mm} \times 20 \mathrm{~mm} \times 4 \mathrm{~mm}$. The upper and lower splints were made of 304 stainless steel with a thickness of $1.5 \mathrm{~mm}$. During the experiment, the sandwich structure was placed inside the shearing box through the holes on both sides and then driven by a servo motor to generate the reciprocating sliding motion against the particles. Two vertical

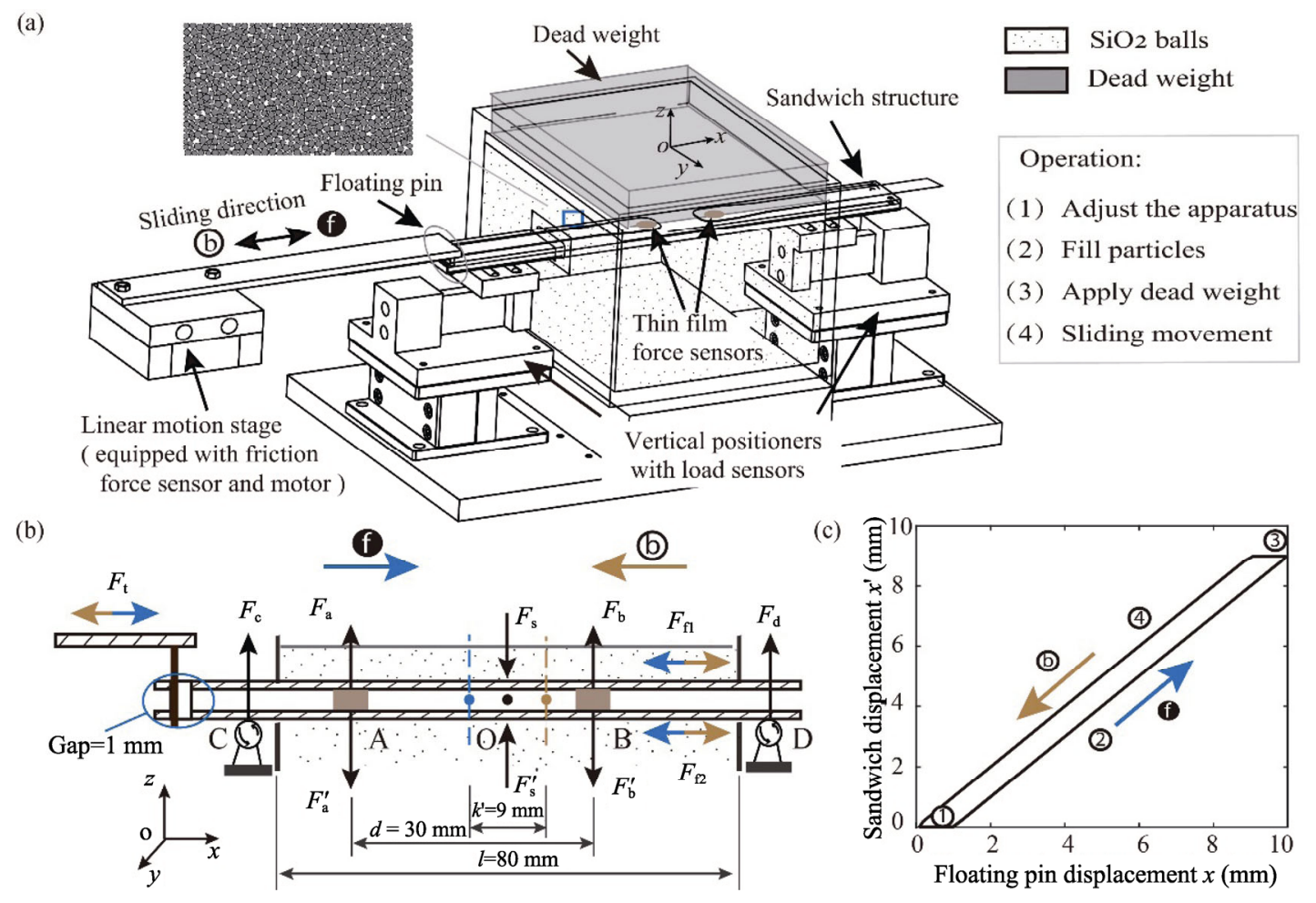

Fig. 1 (a) Schematic diagram of the granular friction apparatus. $\mathbf{f}$ indicates the forward sliding and (b) indicates the reverse sliding. (b) Illustration of the forces acting on the sandwich structure. (c) Displacement of the sandwich structure versus displacement of the floating pin during the reciprocating sliding process. 
position platforms with load cells were placed on both sides of the shearing box to adjust the gap between the sandwich structure and the two holes in the shearing box. The linear motion stage uses a floating pin to drive the sandwich structure to achieve sliding. In this regard, using floating pin satisfies the following requirements: (1) The upper and lower splints of the sandwich structure maintain synchronized horizontal sliding to avoid relative movement with the sensor. (2) The upper and lower splints are not subjected to any additional normal force from the fixture. (3) The experimental apparatus can be assembled and the position of the sandwich structure can be adjusted conveniently. The motion control and data acquisition of the device were realized using LabVIEW, and the data sampling frequency was $1,000 \mathrm{~Hz}$.

\subsection{Experiment operation and motion of sandwich structure}

The procedures conducted during the experiment were as follows: adjusting the apparatus, filling particles, applying a dead weight, performing a reciprocating sliding motion, and recording data. First, the sandwich structure was placed horizontally along the centerline of the holes on the walls by adjusting the vertical position platforms. Subsequently, the particles were filled layer by layer into the shearing box to ensure stable contact between the sandwich structure and particles. Moreover, the top surface of the particle system should remain at a horizontal level. After the filling, a dead weight with almost the same size as the top surface of the shearing box was placed onto the top surface of the particle system to apply an external load uniformly. The external load increased from 1 to 25 $\mathrm{N}$ at an interval of $\Delta G=2.4 \mathrm{~N}$. The actual external load $G^{\prime}$ acting on the sandwich structure accounted for approximately $1 / 5$ of that of the shearing box, as well as approximately $1 / 5$ of the external load $G$. The sliding velocity of the sandwich structure varied from 0.5 to $10 \mathrm{~mm} / \mathrm{s}$ under an external normal load (from 1 to $25 \mathrm{~N}$ ), resulting in an inertial number in the range of $10^{-4}<I<10^{-2}$; hence, we controlled the shear state in the inertial regime.

After loading, the floating pin pushed or pulled the sandwich structure to initiate the reciprocating sliding motion, as shown in Fig. 1(b). A gap of 1.0 $\mathrm{mm}$ was intentionally created between the floating pin and sandwich structure to investigate the behavior of the granular system during the short static period. Therefore, the maximum sliding distances of the floating pin and sandwich structure were 10 and $9 \mathrm{~mm}$, respectively, indicating that the motion of the sandwich structure were lagging behind the floating pin. Owing to this gap, the reciprocating motion of the sandwich structure exhibited the following four stages: stationary (1), forward sliding (2), stationary (3), and reverse sliding (4), as indicated by the corresponding motion curves in Fig. 1(c).

\subsection{Measurement of forces and granular COF}

Six sensors were deployed around the sandwich structure, including two thin-film force sensors (produced by TeKscan, USA) with a measurement range of $0-5 \mathrm{~N}$ and an accuracy of $0.02 \mathrm{~N}$, three capacitive cantilever beam force sensors (produced by HBM, Germany) with a measurement range of 0-20 N and an accuracy of $0.005 \mathrm{~N}$, and one linear variable differential transformer (LVDT) displacement sensor (produced by RDP Group, UK) with a measurement range of $0-25 \mathrm{~mm}$ and an accuracy of $0.025 \mathrm{~mm}$. Thin-film force sensors A and B measured the normal loads on the sandwich structure. The center distance between the two sensors was set to $d=30 \mathrm{~mm}$. The time histories of the positions of the two thin-film force sensors during the reciprocating sliding process were obtained using the displacement sensor. The friction force on the sandwich structure was measured using a cantilever beam force sensor mounted at the left upper end of the reciprocating unit. Cantilever beam force sensors C and D were fixed at the upper ends of the two vertical displacement platforms to measure the supporting normal force $F_{\mathrm{h}}\left(F_{\mathrm{h}}=F_{\mathrm{c}}+F_{\mathrm{d}}\right)$ on the sandwich structure when contact occurred. The forces acting on the sandwich structure are shown in Fig. 1(b). First, the force balance conditions on the upper splint are expressed as follows:

$$
F_{\mathrm{s}}=F_{\mathrm{a}}+F_{\mathrm{b}}
$$




$$
F_{\mathrm{f}}^{\prime}=\mu^{\prime} F_{\mathrm{s}}
$$

where $F_{\mathrm{s}}$ is the normal load from the particles; $F_{\mathrm{a}}$ and $F_{\mathrm{b}}$ are the supporting forces from sensors $\mathrm{A}$ and $\mathrm{B}$, respectively; $F_{\mathrm{f}}^{\prime}$ is the friction force on the surface of the upper splint; $\mu^{\prime}$ is the COF between the upper splint and particles.

For the lower splint, the following conditions are satisfied:

$$
\begin{gathered}
F_{\mathrm{S}}^{\prime}=\left(F_{\mathrm{a}}^{\prime}+F_{\mathrm{b}}^{\prime}\right)-\left(F_{\mathrm{c}}+F_{\mathrm{d}}\right) \\
F_{\mathrm{f}}^{\prime \prime}=\mu^{\prime \prime} F_{\mathrm{S}}^{\prime}
\end{gathered}
$$

where $F_{\mathrm{S}}^{\prime}$ is the normal load from the particles; $F_{\mathrm{f}}^{\prime \prime}$ is the friction force on the lower splint; $\mu^{\prime \prime}$ is the COF between the lower splint and particles; $F_{a}^{\prime}$ and $F_{\mathrm{b}}^{\prime}$ are the reaction forces of $F_{\mathrm{a}}$ and $F_{\mathrm{b}}$, respectively; $F_{\mathrm{c}}$ and $F_{\mathrm{d}}$ are the supporting forces from the vertical displacement platforms.

In this experiment, because the position of the sandwich structure was adjusted to avoid contact with the vertical displacement platform, the measured $F_{\mathrm{c}}$ and $F_{\mathrm{d}}$ were stable and approximately zero; therefore, $F_{\mathrm{c}}+F_{\mathrm{d}}=0$. The measured total friction force $F_{\mathrm{f}}$ is the sum of $F_{\mathrm{f}}^{\prime}$ and $F_{\mathrm{f}}^{\prime \prime}$, namely, $F_{\mathrm{f}}=F_{\mathrm{f}}^{\prime}+F_{\mathrm{f}}^{\prime \prime}=\mu^{\prime} F_{\mathrm{s}}+\mu^{\prime \prime} F_{\mathrm{s}}^{\prime}$. We assume that the granular COF is the same for the top and bottom surfaces, i.e., $\mu=\mu^{\prime}=\mu^{\prime \prime}$, and $F_{\mathrm{s}}=F_{\mathrm{s}}^{\prime}$ when the gravity of the sandwich structure is disregarded. Under these conditions, the relation $F_{\mathrm{f}}=2 \mu F_{\mathrm{s}}$ was obtained. Therefore, the COF between the sandwich structure and particles can be calculated as

$$
\mu=\frac{F_{\mathrm{f}}}{F_{\mathrm{n}}}=\frac{F_{\mathrm{f}}}{2\left(F_{\mathrm{a}}+F_{\mathrm{b}}\right)}
$$

\subsection{Accuracy test of friction apparatus under solid block sliding}

Prior to the granular friction experiment, "solid block sliding" tests, which will be frequently referred to hereinafter, were conducted to verify the accuracy and sensitivity of the measurement forces, where the sandwich structure exhibited reciprocating sliding against two solid acrylic blocks, as illustrated in the subplot of Fig. 2(c). In this case, three weights were used, i.e., 1.77, 2.67, and $3.57 \mathrm{~N}$. As shown in
Fig. 2(a), during the reciprocating sliding, $F_{a}$ and $F_{\mathrm{b}}$ changed periodically and smoothly. Additionally, the measured values of $F_{\mathrm{a}}$ and $F_{\mathrm{b}}$ depended on the positions of the corresponding sensors. As shown in Fig. 2(b), the values of $F_{\mathrm{s}}$ were consistent (error less than 5\%) with the applied weights. In addition, based on the system's moment balance, the following relationship was obtained:

$$
\frac{F_{\mathrm{a}}}{F_{\mathrm{b}}}=\frac{d-k^{\prime}+2 x^{\prime}}{d+k^{\prime}-2 x^{\prime}}
$$

where $x^{\prime}$ is the time history of displacement of the sandwich structure.

It was discovered that the experimental measurement results of $F_{\mathrm{a}} / F_{\mathrm{b}}$ were stable and highly coherent with the calculated values using Eq. (6) (error less than $5 \%$ ), as shown in Fig. 2(c). Therefore, these measurements under solid block sliding demonstrated the high accuracy and reliable sensitivity of the experimental apparatus. In addition, these results can serve as comparison references for the following granular friction.

\section{Results and discussions}

\subsection{Feasibility demonstration of inside-sliding granular friction measurement}

In this section, we present the measurement results of the normal forces, friction force, and COF during the reciprocating sliding process for a granular system. Based on $G=20.2 \mathrm{~N}$ and $v=2 \mathrm{~mm} / \mathrm{s}$ as an example, the experimental time history plots are shown in Fig. 3. Excluding inevitable fluctuations, the measured forces changed periodically during the continuous reciprocating process. However, the evolution patterns of the measured normal forces differed completely with those shown in Fig. 2 because of the discrete nature of the granular system. In detail, in contrast to the linear variation pattern shown in Fig. 2(a), for the granular system, normal forces $F_{\mathrm{a}}$ and $F_{\mathrm{b}}$ alternated between strong and weak in a complicated nonlinear manner, as shown in Fig. 3(a). More importantly, the total normal force $F_{\mathrm{s}}$ shown in Fig. 3(b) did not remain constant but changed regularly. Specifically, $F_{s}$ varied periodically and dynamically between its 

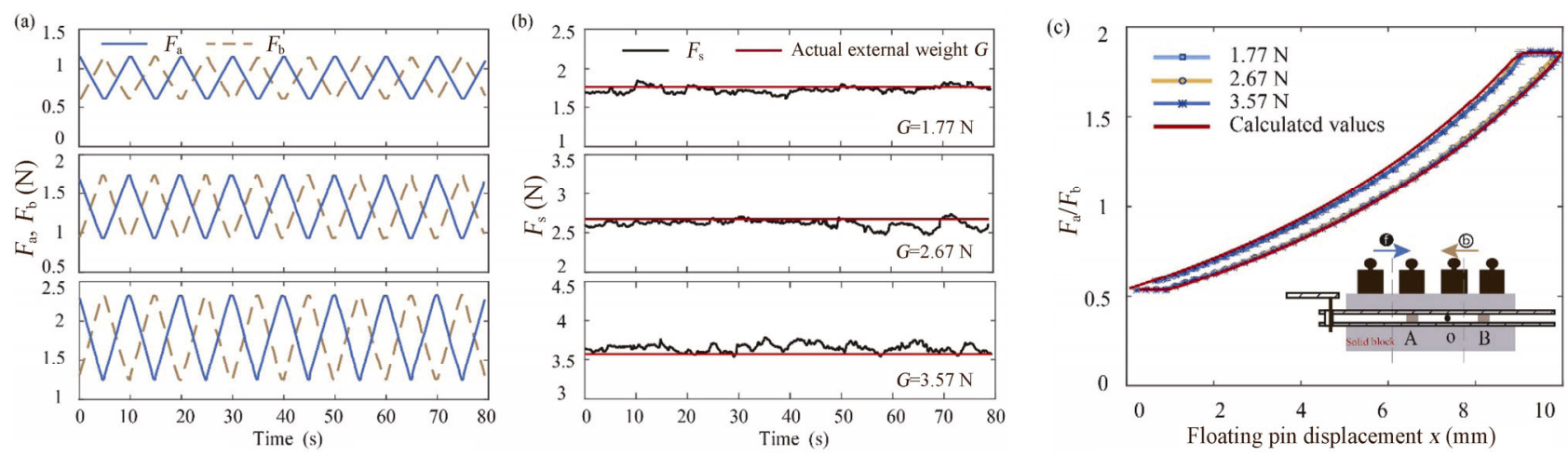

Fig. 2 Accuracy and sensitivity test results: (a) the time history of change curves of $F_{\mathrm{a}}$ and $F_{\mathrm{b}}$; (b) comparison between the experimentally measured normal force $F_{\mathrm{s}}$ and the actual external loading $G$; and (c) $F_{\mathrm{a}} / F_{\mathrm{b}}$ as a function of the floating pin displacement compared with the calculated values, where the subset figure is the structure diagram under solid filling condition.

maximum and minimum values. In this case, its maximum and minimum values were estimated to be 5.0 and $1.0 \mathrm{~N}$, respectively. The total normal force acting on the shearing unit reflects the load-bearing capacity of the granular system. To some extent, the variation in $F_{s}$ can be explained by the reciprocating shearing motion of the sandwich structure, which induces dynamic movements in the particles and changes the force transmission paths, thereby resulting in a variation in the load capacity of the granular system. Furthermore, based on the motion timeline, the four motion stages of the sandwich structure are shown in Fig. 3(e). It was discovered that the measured normal forces changed abruptly with the motion state of the shearing surface; this will be thoroughly discussed

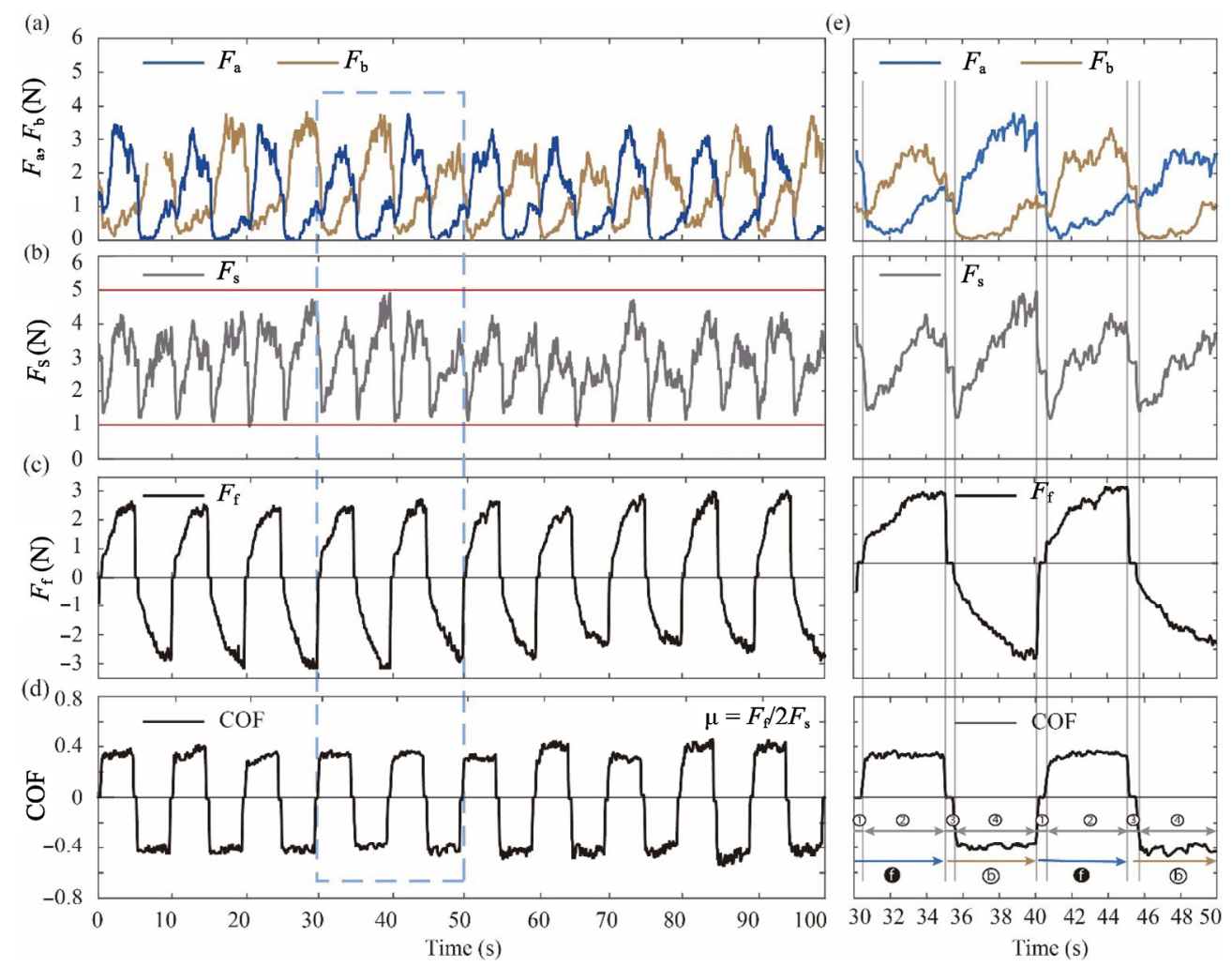

Fig. 3 The experimental time history plots under the conditions of $G=20.2 \mathrm{~N}$ and $v=2 \mathrm{~mm} / \mathrm{s}$, (a)-(d) are the normal forces $F_{\mathrm{a}}, F_{\mathrm{b}}, F_{\mathrm{s}}$, the friction force $F_{\mathrm{f}}$, and COF in 10 cycles respectively, and (e) is the close observations of the two cycles in the blue box, where the four motion stages of the sandwich structure can be observed. 
and explained in the following section.

As shown in Fig. 3(c), the measured friction force $F_{\mathrm{f}}$ varied periodically and smoothly. The change in the sliding direction is indicated by the signs of the friction force $F_{\mathrm{f}}$. Except for the signs, the varying patterns of the friction force $F_{\mathrm{f}}$ curves were almost identical between forward and reverse sliding. As observed, the friction force $F_{\mathrm{f}}$ increased almost monotonically when the shearing unit was moving, and it decreased to zero rapidly at the instant the shearing unit stopped. Based on these measured forces, the granular COF was calculated using Eq. (5) and presented in Fig. 3(d). Despite the dynamic changes in the normal and tangential forces, the COF was relatively stable during sliding, indicating that the $\mathrm{COF}$ is an inherent property of the granular system. Therefore, it is noteworthy that the customized friction apparatus not only successfully measured the time history of the granular COF using the inside-sliding scheme, but also clearly reflected the dynamic load-bearing capacity of the granular system under shearing motion.

\subsection{Effect of shearing motion on evolution behaviors of measured normal forces}

To explain the effect of the motion state of the shearing surface on the evolution behaviors of the measured normal forces, they should be correlated. As previously mentioned, the total normal force acting on the shearing unit is directly determined by the load-bearing capacity of the granular system, which is typically characterized by force chain. Therefore, the force chain can be used for the correlation. Moreover, according to a previous study [40], during a reciprocating sliding process with an abrupt change in the moving direction, the force chain experiences the following four stages: relaxation, collapse, reconstruction, and stability. Hence, to more clearly identify the motion stages of the shearing unit, the force-time curves were converted into force-displacement plots. Furthermore, the displacement of the floating pin was used instead of that of the sandwich structure such that the granular system's response at the moment the sandwich structure became stationary can be reflected. It is noteworthy that, as indicated by Eq.
(6) and Fig. 2(a), even for the solid block sliding, the measured forces $F_{\mathrm{a}}$ and $F_{\mathrm{b}}$ were dependent on the position of the sandwich structure as a result of the system's force and moment balance. In terms of sliding inside the granular matter, the dependence of $F_{\mathrm{a}}$ and $F_{\mathrm{b}}$ on position will be much more complex because of the dynamic evolution of the force chain network. Additionally, their sum $F_{s}$ will no longer remain constant, which differs from the case of solid block sliding.

In the following paragraphs, we will fully explain the experimental force measurement results based on the three sequential stages marked in Fig. 4(a), i.e., the first stationary stage (positions $P_{1}$ to $P_{2}$ ), the initial forward sliding (position $P_{2}$ to $P_{3}$ ), and the subsequent sliding (position $P_{3}$ to $P_{4}$ ). In addition, to understand the underlying reasons for the changes in the measured forces more effectively, images illustrating the topology and strength of the force chain (Fig. 4(f)) during the reciprocating sliding process are depicted at the aforementioned four positions. It is noteworthy that these force chain images were reasonably inferred based on the experimental results and the conclusions in Ref. [40], albeit only for the qualitative explanation provided herein. More simulations pertaining to the force chain for our case will be conducted in the future.

As shown in Figs. 4(a)-4(d), in the first stationary stage (displacement range of $0-1 \mathrm{~mm}$ ), the sandwich structure completes the reverse sliding motion and enters the static state. During this stage, the system does not bear the external shearing motion, which weakens the constraint between particles, and the strong force chains (position $P_{1}$ in Fig. 4(f)) relaxes to a weak one (position $P_{2}$ in Fig. 4(f)). Because of the relaxation of the force chains, the load bearing capacity of the granular system is significantly weakened; therefore, normal forces $F_{\mathrm{a}}, F_{\mathrm{b}}$, and $F_{\mathrm{s}}$ decrease significantly. Because $F_{\mathrm{a}}$ decreases more quickly than $F_{\mathrm{b}}$, the ratio $F_{\mathrm{a}} / F_{\mathrm{b}}$ is reduced significantly. Meanwhile, the friction force $F_{f}$ reduces to zero abruptly, similar to the COF. Furthermore, a significant difference in $F_{\mathrm{a}} / F_{\mathrm{b}}$ was observed between the granular friction and solid block friction, as shown in Fig. 4(d). For the solid block sliding, $F_{\mathrm{a}} / F_{\mathrm{b}}$ 

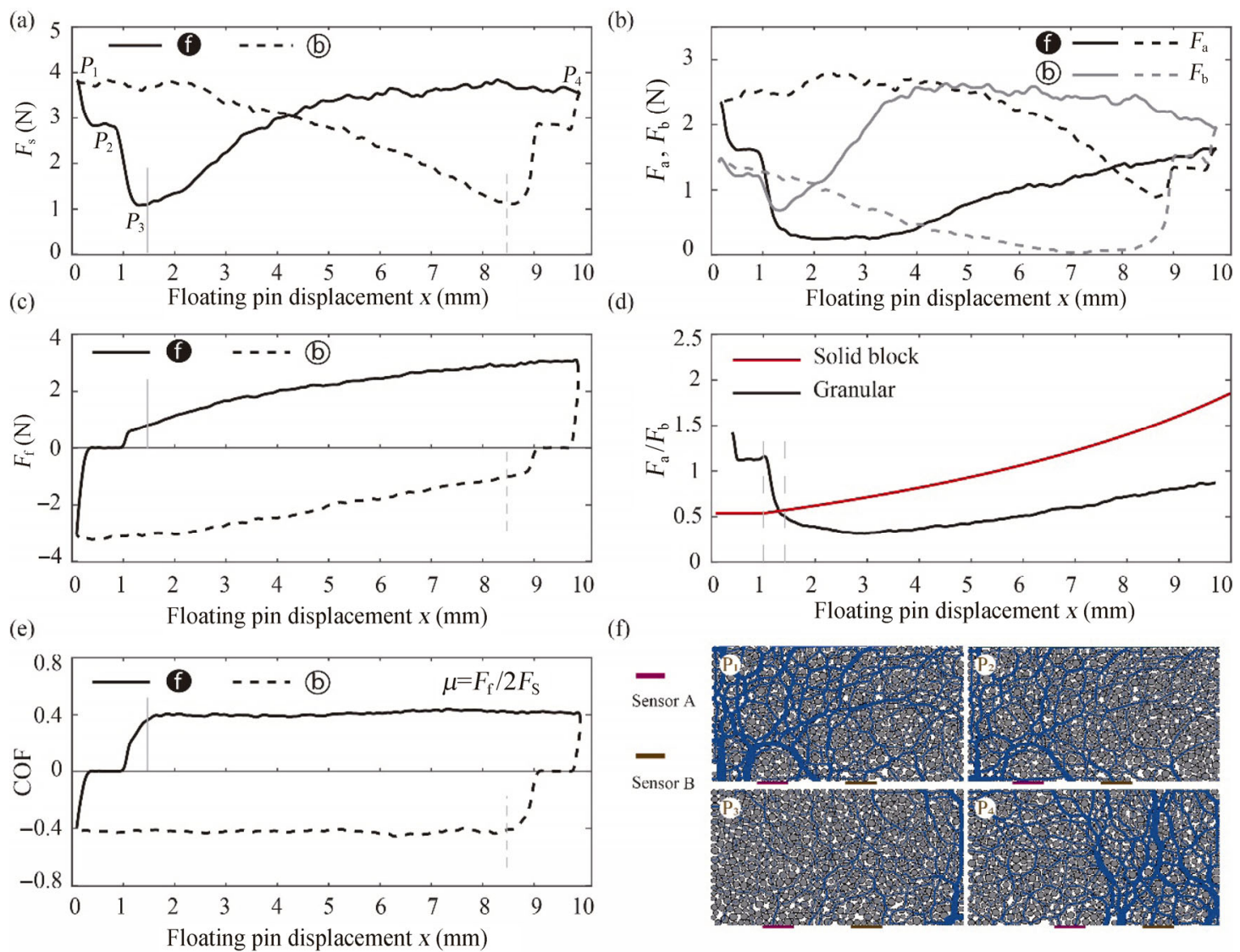

Fig. 4 The evolution behaviors of $F_{\mathrm{s}}(\mathrm{a}), F_{\mathrm{a}}$ and $F_{\mathrm{b}}$ (b), $F_{\mathrm{f}}$ (c), $F_{\mathrm{a}} / F_{\mathrm{b}}$ (d), and COF (e) with regards to the floating pin displacement. (f) Images for illustrating the topology and strength of the force chain at four special positions $\left(P_{1}\right.$ to $P_{4}$ in (a)).

remained constant at approximately 0.50; however, the value was much greater for the granular system. This is probably caused by the fact that immediately after the reverse sliding, the force chains primarily reside on or tilt to the left side (as illustrated by position $P_{1}$ in Fig. 4(f)); consequently, the load-bearing capacity on the left side becomes stronger, resulting in a greater $F_{\mathrm{a}} / F_{\mathrm{b}}$. After the relaxation, all the measured forces remained steady, indicating that the system was stable for a short time.

During forward sliding, two remarkable stages can be distinguished based on the evolution trends of the force curves. In detail, in the initial sliding stage (displacement range of $1-1.5 \mathrm{~mm}$ ), the granular system began to sustain a shear force in the direction opposite to the previous reverse sliding. At this moment, because the force chains were weakened severely (as illustrated by position $P_{3}$ in Fig. 4(f)) after the first stationary stage, the system's ability to resist the opposite shearing motion was extremely weak, thereby resulting in an instantaneous collapse of the force chains. Therefore, all normal forces were abruptly reduced to a minimum. Similarly, $F_{a}$ decreased more quickly than $F_{\mathrm{b}}$, and $F_{\mathrm{a}}$ became lower than $F_{\mathrm{b}}$ at a certain moment. Consequently, the $F_{\mathrm{a}} / F_{\mathrm{b}}$ for the granular system decreased significantly and became less than that of the solid block sliding. These observations indicate that the effect of the collapse of the force chain was more evident on the left side. During the initial sliding stage, the friction force $F_{\mathrm{f}}$ increased gradually. Meanwhile, the COF increased quickly and almost reached the stable value of the system at the end.

In the following sliding (displacement range of $1.5-10 \mathrm{~mm}$ ), the force chains were dynamically reorganized and stabilized gradually because of the constraints of both the load and spatial boundary conditions. By comparing the evolution 
behaviors of these normal forces between the granular system and solid block sliding, significant differences were observed, and the underlying effect induced by the force chain was identified. Specifically, for solid block sliding, the normal force $F_{\mathrm{s}}$ remained constant. By contrast, for the granular system, $F_{\mathrm{s}}$ increased gradually before reaching stability owing to the reconstruction stabilization of the force chains [24]. The friction force $F_{\mathrm{f}}$ varied in a pattern similar to that of $F_{\mathrm{s}}$. Moreover, for the solid block sliding, $F_{\mathrm{b}}$ continuously decreased during forward sliding. However, for the granular system, $F_{\mathrm{b}}$ increased gradually initially, stabilized in the middle range, and decreased slightly decreased at the end. In addition, during this stage, $F_{\mathrm{b}}$ was larger than $F_{\mathrm{a}}$. Correspondingly, the ratio $F_{\mathrm{a}} / F_{\mathrm{b}}$ for the granular system increased gradually, but its rate of increase was less than that of the solid block sliding. Based on these observations, it can be conjectured that during this forward sliding stage, the force chain primarily regenerated on or inclined to the right side (as illustrated by position $P_{4}$ in Fig. 4(f)), thereby resulting in the peculiar behavior of $F_{b}$.

After the forward sliding, the granular system entered the second stationary stage and then underwent reverse sliding. The measured results were similar to those of forward sliding, and the principles and explanations above can be applied. At the end of the reverse sliding, the force chain primarily resided on or tilted to the left side; as such, $F_{\mathrm{a}}$ was greater than $F_{\mathrm{b}}$ at the exact beginning moment of the first stationary stage.

In summary, the evolution behaviors of the measured normal forces and friction forces were presented herein within a reciprocating loop. Comprehensive discussions were provided to correlate the evolution behaviors of the normal forces and the evolution of the force chain network within the granular system. The spatial distribution and dynamic change of the force chains were implicitly reflected by the peculiar evolution behaviors of $F_{\mathrm{a}}$ and $F_{\mathrm{b}}$. During the half-loop period of reciprocating sliding, the normal force $F_{s}$ underwent four typical stages: an abrupt decrease followed by a momentary stabilization, a further significant decrease to the minimum, a gradual increase to the maximum, and finally stabilization. These stages were associated closely to the relaxation, collapse, reconstruction, and stabilization of the force chain, respectively. Correspondingly, the COF decreased significantly to zero during the stationary stage, increased to a stable value during the initial sliding, and then remained almost constant during the following sliding stage.

\subsection{Effect of external load on granular friction}

In granular friction, the external load and sliding velocity are two key factors affecting the COF [8, $15,41]$. Based on sliding velocity $v=2 \mathrm{~mm} / \mathrm{s}$ as an example, the effects of the external load were investigated. During the experiment, the sliding velocity was fixed, whereas the external loads were linearly increased from 1 to $25 \mathrm{~N}$ during the trials. The measured normal force, friction force, and COF are shown in Fig. 5(a). It was evident that the maximum amplitudes of the measured normal and friction forces increased with the external loads. However, the amplitude of the COF decreased gradually, which directly reflected the dependence of the COF on the external load.

Because the discrete nature of granular matter caused inevitable fluctuations in the measurement results, the average values of the measured results within 20 cycles were plotted against the sliding displacement of the floating pin, as shown in Figs. 5(b)-5(d). It was discovered that all the measured results under different loads exhibited similar patterns. However, when the external load was small (e.g., $G=1 \mathrm{~N}$ ), the friction force was much larger than the normal force. As the external load increased, the friction force gradually approached the normal force and finally became less than that when the external load was large. Correspondingly, the stable COF decreased significantly with the external load, from much greater than 1.0 at a small load to less than 1.0 at a large load, as shown in Fig. 5(d).

In the motion range of $1.5-10 \mathrm{~mm}$ during forward sliding and $8.5-0 \mathrm{~mm}$ during reverse sliding, the COF fluctuated slightly. Therefore, the average $\mathrm{COF}$ values in these ranges were defined as the stable $\mathrm{COF}, \bar{\mu}$. Furthermore, to quantitatively 

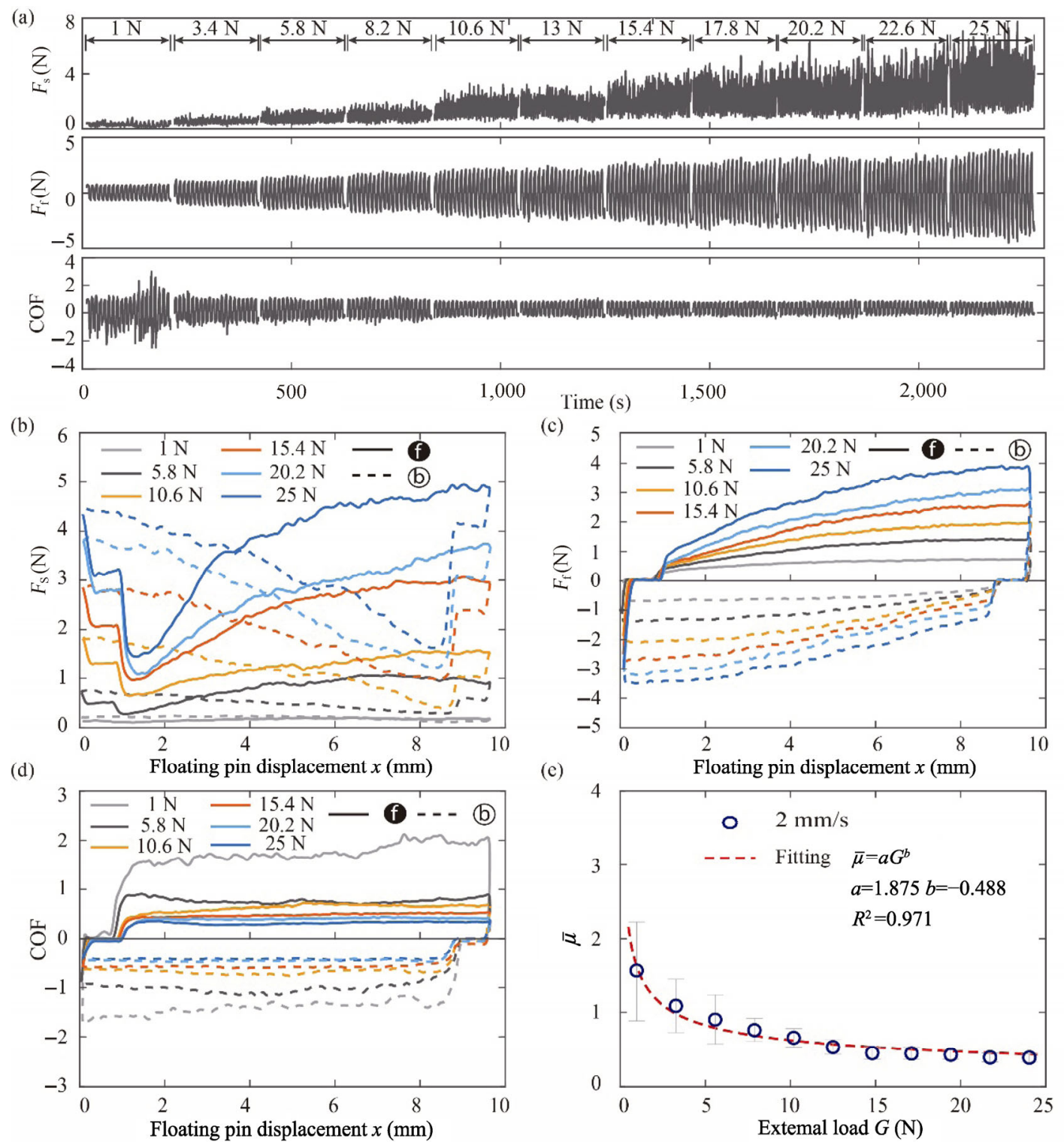

Fig. 5 Effect of the external load on the granular friction behaviors under sliding velocity $v=2 \mathrm{~mm} / \mathrm{s}$. (a) Time history plots of the measurement results. (b), (c), and (d) are the average values of $F_{\mathrm{s}}, F_{\mathrm{f}}$, and COF in 20 cycles versus the floating pin displacement respectively. (e) The evolution curve of the stable COF $\bar{\mu}$ as a function of the external load.

investigate the dependence of the COF on the external load, the stable COF values were plotted against the external load, as shown in Fig. 5(e). It was discovered that the stable COF decreased significantly with the external load in a powerfunction form, i.e., $\bar{\mu}=a G^{b}(a=1.875, \quad b=-0.488)$ with good confidence.

Similarly, the dependence of the COF on the external load was further investigated for all the sliding velocities tested. As shown in Fig. 6(a), for different sliding velocities (from 0.5 to $10 \mathrm{~mm} / \mathrm{s}$ ), all the stable COFs against the external load were well fitted using the power function, $\bar{\mu}=a G^{b}$. Subsequently, the fitting parameters were analyzed to reveal more in-depth information. First, parameter $a$, which is similar to the maximum measured COF at a certain sliding velocity, indicated an overall increasing trend with the sliding velocity, as shown in Fig. 6(b). More importantly, the fitting coefficient $b$, which is the decaying extent, fluctuated slightly around -0.5 for all the cases, as shown in Fig. 6(c). This result indicates that the 

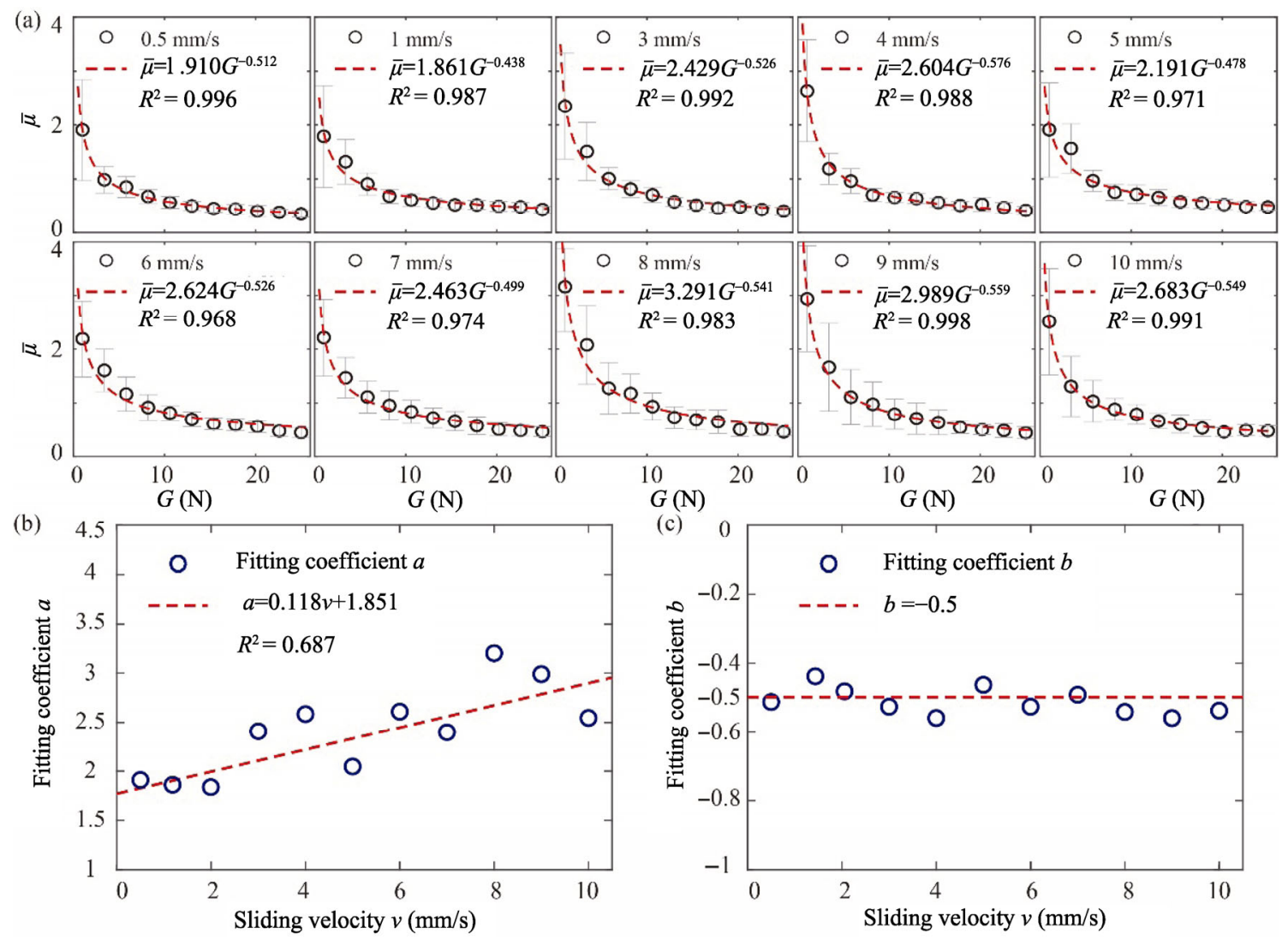

Fig. 6 (a) Plots of the stable COF versus the external load at all tested sliding velocities. (b) and (c) Trend of the fitting parameters $a$ and $b$ as a function of the sliding velocity respectively.

granular COF is intrinsically affected by the external load. Our experimental findings are consistent with those of previous studies. For example, in Refs. [8, 25, 26], the granular COF was reported to be associated closely with the inertial number $I$, as follows:

$$
\mu(I)=\mu_{\mathrm{c}}+a_{\mu} I=\mu_{\mathrm{c}}+a_{\mu} \dot{\gamma} \frac{d}{\sqrt{P / \rho}}
$$

where $\mu_{\mathrm{c}}$ denotes the critical value at zero shear rate, $a_{\mu}$ a constant, $\dot{\gamma}$ the sliding rate, $P$ the normal stress, $d$ the particle diameter, and $\rho$ the particle density. This equation indicates that the COF is dependent of the normal stress $P$ in the mathematical form of $P^{-0.5}$, as confirmed in our experimental findings.

\subsection{Effect of sliding velocity on granular friction}

Similarly, under a fixed external load, the sliding velocity was increased from 0.5 to $10 \mathrm{~mm} / \mathrm{s}$ to analyze the effect of sliding velocity on granular friction. Based on the external load $G=20.2 \mathrm{~N}$ as an example, the time history plots of the measured results were constructed, as presented in Fig. 7(a). As shown, the normal and friction forces fluctuated slightly, and the COF did not change significantly with the sliding velocity. Hence, compared with the external load, the effect of the sliding velocity on the measurement results was slight.

Subsequently, the averaged measurement results for 20 cycles were plotted against the sliding displacement of the floating pin. As shown in Figs. $7(\mathrm{~b})-7(\mathrm{~d})$, the curves under different sliding velocities exhibited similar patterns. However, as shown in Fig. 7(d), a greater sliding velocity necessitated a greater displacement for the COF to attain a stable value. Finally, the dependence of the stable COF on the sliding velocity is presented in Fig. 7(e). As shown, the stable COF increased slightly with the sliding velocity from the overall trend, and this can be fitted using the linear equation $\bar{\mu}=k v+c \quad(k=0.0226, c=0.3746)$. 

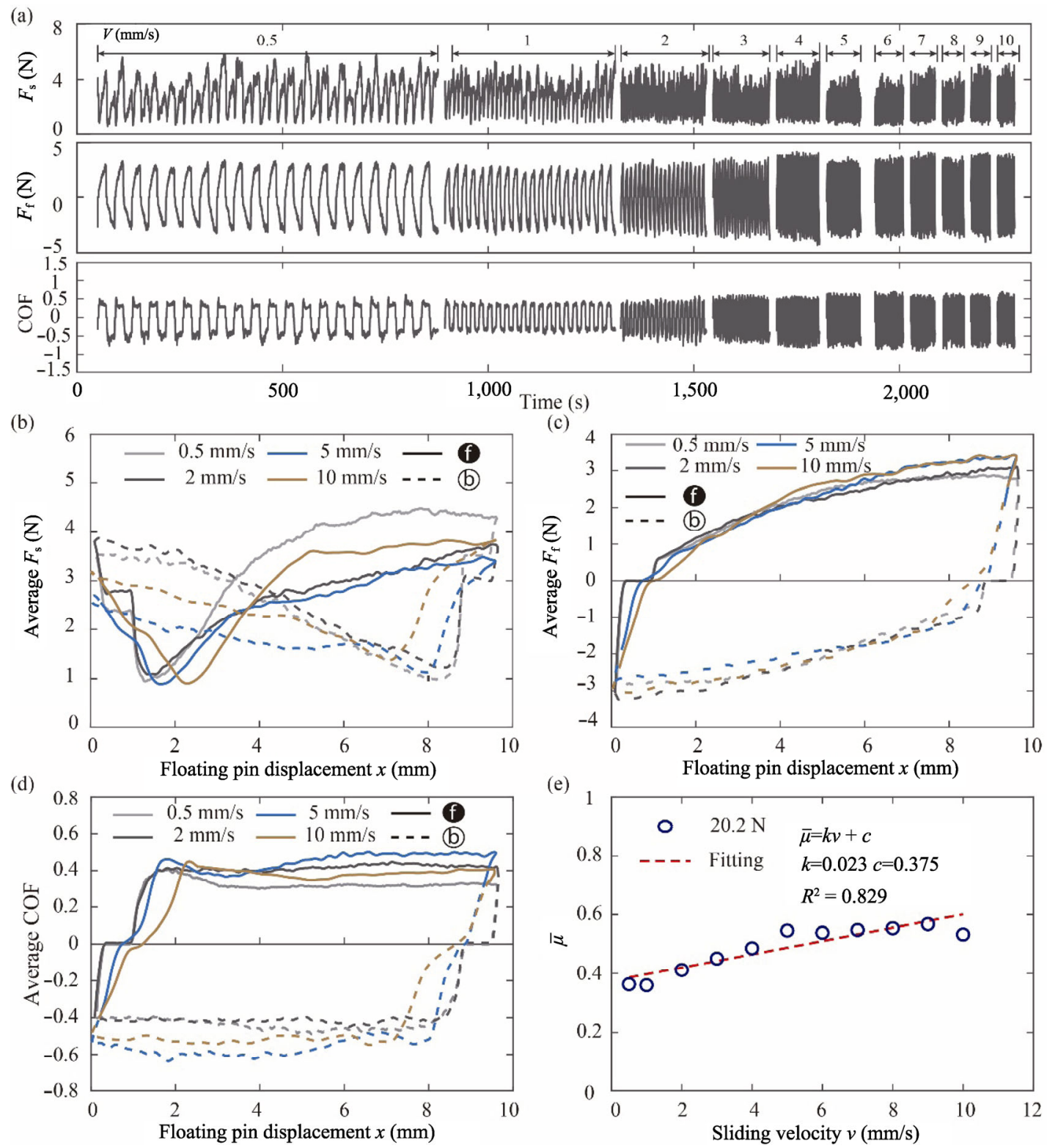

Fig. 7 Effect of sliding velocity on the granular friction behaviors under external load $G=20.2$ N. (a) Time history plots of the measured normal force $F_{\mathrm{s}}$, friction force $F_{\mathrm{f}}$, and COF. (b), (c), and (d) are the average values of $F_{\mathrm{s}}, F_{\mathrm{f}}$, and COF in 20 cycles versus the floating pin displacement respectively. (e) The change of the stable COF $\bar{\mu}$ as a function of the sliding velocity.

Furthermore, the dependence of the average $\mathrm{COF}$ on the sliding velocity was investigated for all the tested external loads, as shown in Fig. 8. As shown in these plots, although the average COF fluctuated with the sliding velocity, an overall increasing trend was observed. Based on Eq. (7), the average COF increases linearly with the shear rate. Typically, the shear rate within a granular system is proportional to the sliding velocity of the shearing unit. Therefore, the stable COF should increase with the sliding velocity in our experiments. Additionally, the curves in Fig. 8(a) were fitted using the linear equation $\bar{\mu}=k v+c$. As the external load increased, both the fitting slope $k$ and constant $c$ decreased with the external load in a power-function form, as shown in Figs. 8(b)-8(c). The decrease in slope $k$ indicates that the degree of dynamic change induced by the shearing motion weakened gradually. Meanwhile, the decrease in the fitting constant $c$, which represents the COF at 
an extremely small sliding velocity, indicates improved system stability.

Finally, an overall illustration of the stable COF's dependence on the external load and sliding velocity was provided, as shown by the results of $\bar{\mu}$ and standard deviations of $\bar{\mu}$ in Figs. 9(a) and $9(\mathrm{~b})$, respectively. As shown in Fig. 9(a), the experimental stable COF results were well fitted using a formula containing both the external load $G$ and sliding velocity $v$, in the form of $\bar{\mu}=(k v+c) G^{b}$, where $k=0.089, c=2.193$, and $b=$ -0.556 . It was confirmed that the granular COF decreased with the external load in a form similar to the power-function form of $G^{-0.5}$. Furthermore, two side views of Fig. 9(a) were presented in Figs. 9(c) and 9(d) to show the dependences of the COF more clearly. As shown in Figs. 9(a) and 9(c), under small external loads, the COFs were large (primarily greater than 1.0), and they fluctuated significantly, as indicated by the considerable standard deviations. As the external load increased, $\bar{\mu}$ decreased significantly, and the standard deviations decreased as well. By contrast, as shown in Fig. 9(d), because the range of the sliding velocity was relatively narrow, the COF only increased slightly with the sliding velocity. Additionally, by comparing the fitting confidence values in Figs. 6(a) and 8(a), it was discovered that the power-law dependence of the COF on the external load was significant, whereas the linear dependence of the COF on the sliding velocity was relatively weak. Because the range of the sliding velocity was relatively narrow compared with that reported in previous studies $[15,27,41]$, the COF only increased slightly with the sliding velocity. Therefore, in this study, the COFs were much more sensitive to the external load than to the sliding velocity. This highlights the advantage of the inside-sliding scheme in reflecting the effect of the external load on the granular friction behavior. Moreover, as shown in Fig. 9(d), in a case involving a low load and a high velocity, the inertial number was relatively
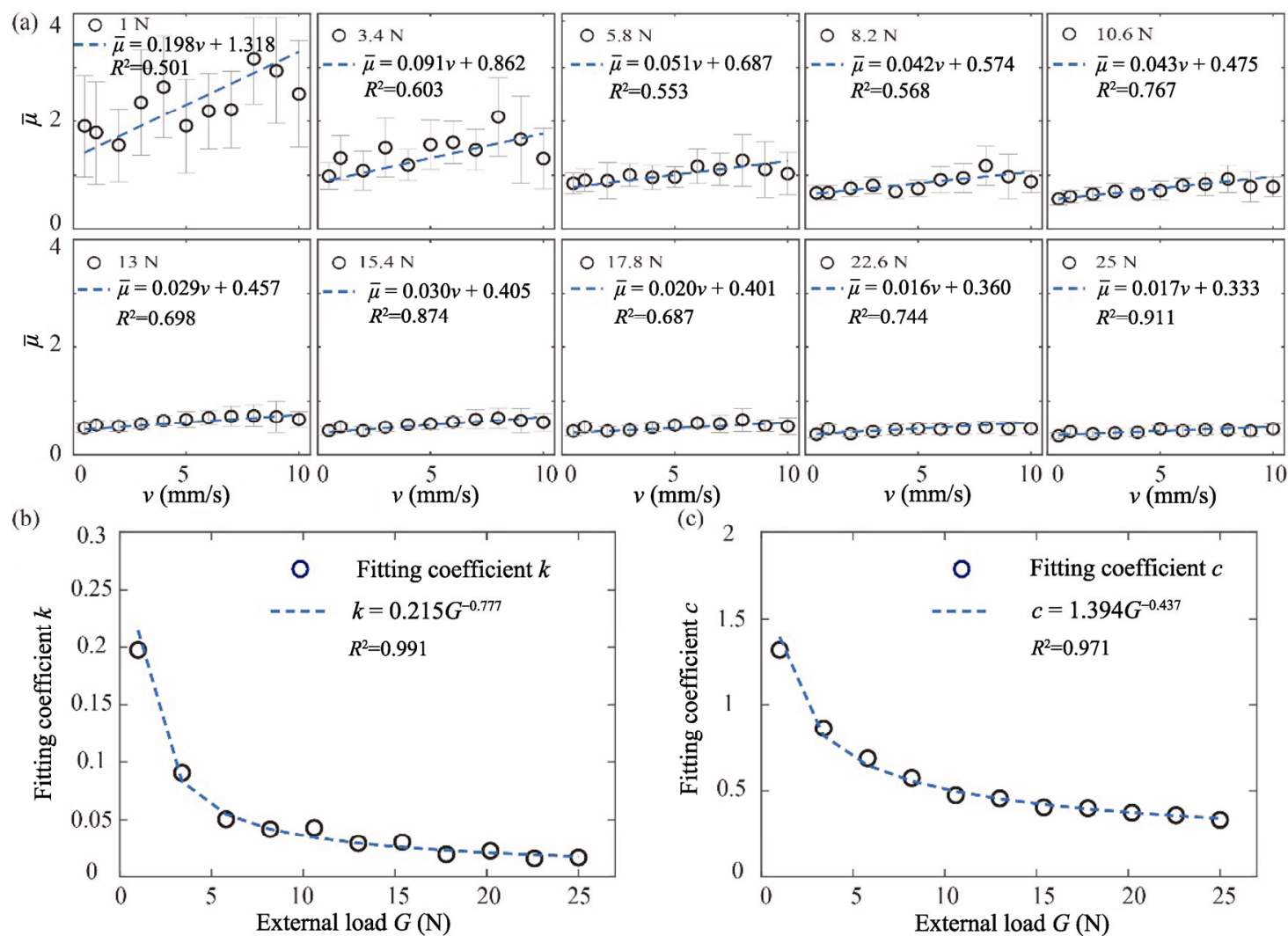

Fig. 8 (a) The stable COF versus sliding velocity at all tested external loads. (b) and (c) Trend of the fitting slope $k$ and the fitting constant $c$ as a function of the external load respectively. 
(a)

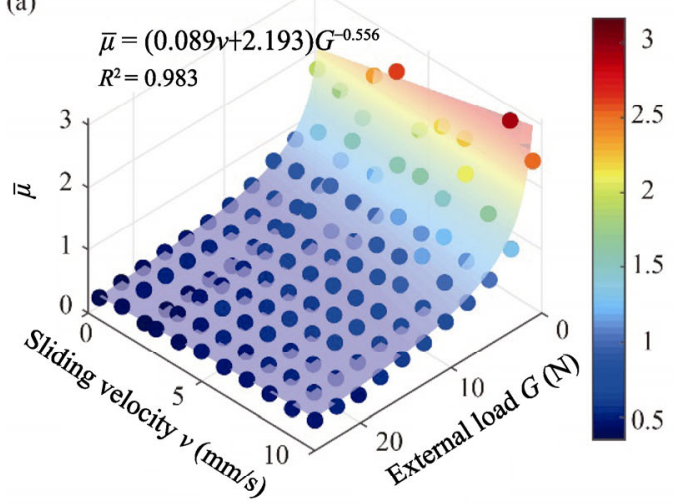

(c)

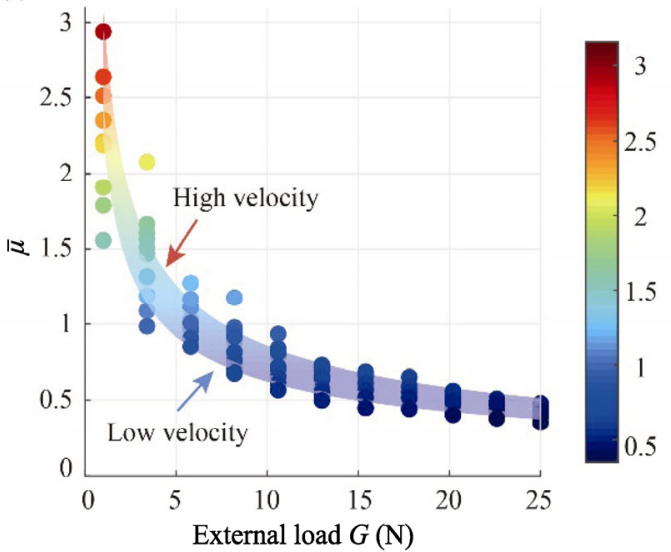

(b)

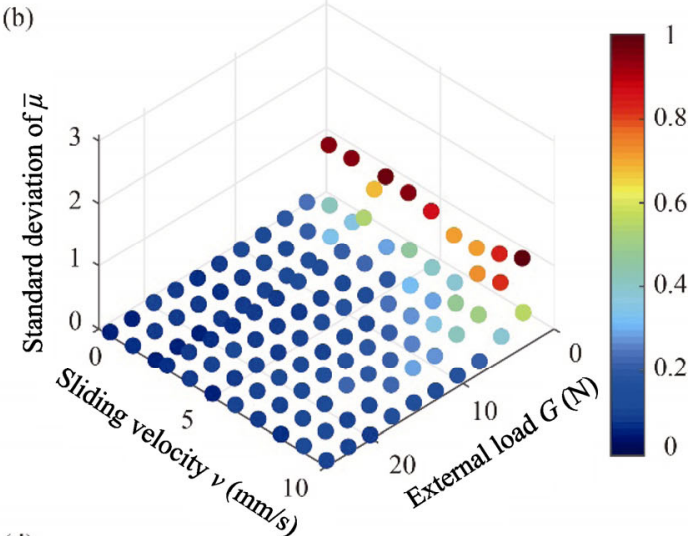

(d)

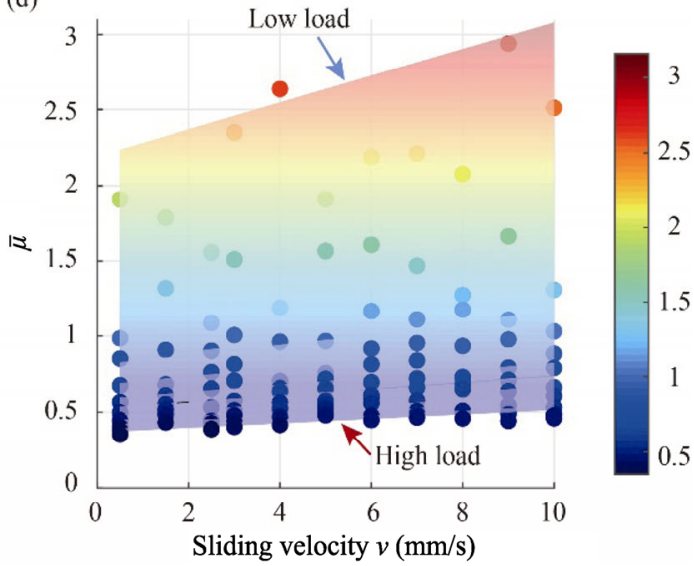

Fig. 9 Dependences of the stable COF $\bar{\mu}$ on external load and sliding velocity. (a) The experimental results of $\bar{\mu}$ which are marked using solid circles, and the fitting formula is plotted using color contour. (b) The standard deviations of $\bar{\mu}$ determined from experiments. (c) and (d) are the side views of (a) to better show the dependences of $\bar{\mu}$ on external load and sliding velocity respectively.

large, resulting in a high and unstable COF. However, in a case involving a high load and a low velocity, the inertial number was relatively small, resulting in a small and stable COF. These experimental findings were highly coherent with granular friction theories reported in Refs. [13, 26].

\section{Conclusions}

The granular friction behavior during a linearly reciprocating sliding process was experimentally investigated using a customized granular friction apparatus. During the half-loop of the reciprocating sliding, the normal force acting on the shearing unit underwent four typical stages: decreased abruptly and stabilized momentarily, further decreased significantly to the minimum, gradually increased to the maximum, and then remained unchanged. These stages were associated closely with the relaxation, collapse, reconstruction, and stabilization of the force chain network, respectively. Correspondingly, the COF decreased to zero during the stationary stage, increased to a stable value during the initial sliding, and then remained almost constant during the following sliding.

Furthermore, the effects of the external load and sliding velocity on the granular friction behaviors were analyzed. It was discovered that the average COF during the stable sliding range decreased significantly with the external load $G$ in the power-function form of $G^{-0.5}$. Additionally, the COF under a small load was extremely large and unstable. Meanwhile, the COF increased slightly with the sliding velocity. Therefore, the COF was more sensitive to the external load than to the sliding velocity. Finally, a complete illustration of the COF's dependence on the external load and sliding velocity was provided. 
This study provides an innovative experimental approach for directly measuring the granular COF and implicitly reflecting the evolution of the force chain network, thereby potentially benefitting granular friction research. In future studies, the sliding velocity range will be expanded and the microscopic dynamic change of particles quantified numerically to further investigate the bearing and friction characteristics of granular matter.

\section{Acknowledgement}

This work was financially supported by the National Natural Science Foundation of China (Nos. 51975174 and 51875154).

Open Access This article is licensed under a Creative Commons Attribution 4.0 International License, which permits use, sharing, adaptation, distribution and reproduction in any medium or format, as long as you give appropriate credit to the original author(s) and the source, provide a link to the Creative Commons licence, and indicate if changes were made.

The images or other third party material in this article are included in the article's Creative Commons licence, unless indicated otherwise in a credit line to the material. If material is not included in the article's Creative Commons licence and your intended use is not permitted by statutory regulation or exceeds the permitted use, you will need to obtain permission directly from the copyright holder.

To view a copy of this licence, visit http://creativecommons.org/licenses/by/4.0/.

\section{References}

[1] Anthony J L, Marone C. Influence of particle characteristics on granular friction. J Geophys Res Solid Earth 110(8): B08409 (2005)

[2] Lherminier S, Planet R, dit Vehel V L, Simon G, Vanel L, Måløy K J, Ramos O. Continuously sheared granular matter reproduces in detail seismicity laws. Phys Rev Lett 122(21): 218501 (2019)

[3] Zhou J, Zhu C Y, Zhang W, Ai W T, Zhang X J, Liu K. Experimental and 3D MPFEM simulation study on the green density of $\mathrm{Ti}-6 \mathrm{Al}-4 \mathrm{~V}$ powder compact during uniaxial high velocity compaction. $J$ Alloys Compd 817: 153226 (2020)
[4] Heshmat H. The rheology and hydrodynamics of dry powder lubrication. Tribol Trans 34(3): 433-439 (1991)

[5] Jang J T, Khonsari M M. On the granular lubrication theory. Proc R Soc London, Ser A 461(2062): 3255 3278 (2005)

[6] Berger N, Azéma E, Douce J F, Radjai F. Scaling behaviour of cohesive granular flows. Europhys Lett 112(6): 64004 (2015)

[7] Nakatani M. Conceptual and physical clarification of rate and state friction: Frictional sliding as a thermally activated rheology. J Geophys Res Solid Earth 106(B7): 13347-13380 (2001)

[8] Jop P, Forterre Y, Pouliquen O. A constitutive law for dense granular flows. Nature 441(7094): 727-730 (2006)

[9] Binaree T, Azéma E, Estrada N, Renouf M, Preechawuttipong I. Combined effects of contact friction and particle shape on strength properties and microstructure of sheared granular media. Phys Rev E 102(2): 022901 (2020)

[10] Nasuno S, Kudrolli A, Gollub J P. Friction in granular layers: Hysteresis and precursors. Phys Rev Lett 79(5): 949-952 (1997)

[11] Nasuno S, Kudrolli A, Bak A, Gollub J P. Time-resolved studies of stick-slip friction in sheared granular layers. Phys Rev E 58(2): 2161-2171 (1998)

[12] Géminard J C, Losert W. Frictional properties of bidisperse granular matter: Effect of mixing ratio. Phys Rev E 65(4): 041301 (2002)

[13] MiDi G. On dense granular flows. Euro Phys $J$ E 14(4): 341-365 (2004)

[14] Siavoshi S, Orpe A V, Kudrolli A. Friction of a slider on a granular layer: Nonmonotonic thickness dependence and effect of boundary conditions. Phys Rev E 73(1): 010301 (2006)

[15] Kuwano O, Ando R, Hatano T. Crossover from negative to positive shear rate dependence in granular friction. Geophys Res Lett 40(7): 1295-1299 (2013)

[16] Horváth V K, Jánosi I M, Vella P J. Anomalous density dependence of static friction in sand. Phys Rev E 54(2): 2005-2009 (1996)

[17] Knuth M, Marone C. Friction of sheared granular layers: Role of particle dimensionality, surface roughness, and material properties. Geochem Geophys Geosyst 8(3): Q03012 (2007)

[18] Peyneau P E. Etude du comportement et du compactage de pates granulaires par simulation numerique discrete. (in French). Ph.D. Thesis. Ecole des Ponts ParisTech, 2009.

[19] Fall A, Weber B, Pakpour M, Lenoir N, Shahidzadeh N, Fiscina J, Wagner C, Bonn D. Sliding friction on wet and dry sand. Phys Rev Lett 112(17): 175502 (2014)

[20] Azéma E, Preechawuttipong I, Radjai F. Binary mixtures of disks and elongated particles: Texture and mechanical properties. Phys Rev E 94(4): 042901 (2016) 
[21] Daniels K E, Kollmer J E, Puckett J G. Photoelastic force measurements in granular materials. Rev Sci Instrum 88(5): 051808 (2017)

[22] Hurley R C, Hall S A, Andrade J E, Wright J. Quantifying interparticle forces and heterogeneity in 3D granular materials. Phys Rev Lett 117(9): 098005 (2016)

[23] Mueth D M, Debregeas G F, Karczmar G S, Eng P J, Nagel S R, Jaeger H M. Signatures of granular microstructure in dense shear flows. Nature 406(6794): 385-389 (2000)

[24] Schaeffer D G, Barker T, Tsuji D, Gremaud P, Shearer M, Gray J M N T. Constitutive relations for compressible granular flow in the inertial regime. J Fluid Mech 874: 926-951 (2019)

[25] Iordanoff I, Khonsari M M. Granular lubrication: Toward an understanding of the transition between kinetic and quasi-fluid regime. $J$ Tribol 126(1): 137-145 (2004)

[26] Da Cruz F, Emam S, Prochnow M, Roux J N, Chevoir F. Rheophysics of dense granular materials: Discrete simulation of plane shear flows. Phys Rev E 72(2): 021309 (2005)

[27] Hatano T. Power-law friction in closely packed granular materials. Phys Rev E 75(6): 060301 (2007)

[28] Ovarlez G, Kolb E, Clément E. Rheology of a confined granular material. Phys Rev E 64(6): 060302 (2001)

[29] Sun Q C, Jin F, Liu J G, Zhang G H. Understanding force chains in dense granular materials. Int J Mod Phys B 24(29): 5743-5759 (2010)

[30] Wang W, Gu W, Liu K. Force chain evolution and force characteristics of shearing granular media in Taylor-

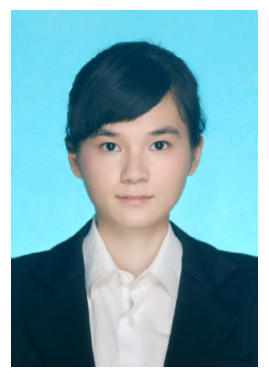

Xuejie ZHANG. She received her bachelor and M.S. degrees in vehicle engineering from Anhui University of Technology, China, in 2013 and 2016, respectively. Now, she is a

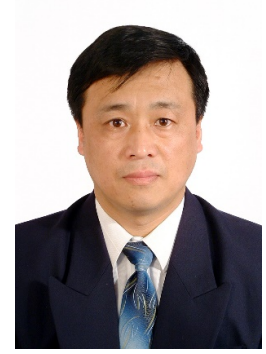

Kun LIU. He received his M.S. degree in 1988 from Hefei University of Technology, Hefei, China, and received his Ph.D. degree in Institute of Lubrication Theory and Bearings in 1995 from Xi'an Jiaotong University, Xi'an,
Couette geometry by DEM. Tribol Trans 58(2): 197-206 (2015)

[31] Mueth D M, Jaeger H M, Nagel S R. Force distribution in a granular medium. Phys Rev E 57(3): 3164-3169 (1998)

[32] Snoeijer J H, Vlugt T J H, van Hecke M, van Saarloos W. Force network ensemble: A new approach to static granular matter. Phys Rev Lett 92(5): 054302 (2004)

[33] Majmudar T S, Behringer R P. Contact force measurements and stress-induced anisotropy in granular materials. Nature 435(7045): 1079-1082 (2005)

[34] Howell D, Behringer R P, Veje C. Stress fluctuations in a 2D granular Couette experiment: A continuous transition. Phys Rev Lett 82(26): 5241-5244 (1999)

[35] Kuhn M R. Structured deformation in granular materials. Mech Mater 31(6): 407-429 (1999)

[36] Jop P. Rheological properties of dense granular flows. $C$ $R$ Phys 16(1): 62-72 (2015)

[37] Divoux T, Géminard J C. Friction and dilatancy in immersed granular matter. Phys Rev Lett 99(25): 258301 (2007)

[38] Cassar C, Nicolas M, Pouliquen O. Submarine granular flows down inclined planes. Phys Fluids 17(10): 103301 (2005)

[39] Azéma E, Linero S, Estrada N, Lizcano A. Shear strength and microstructure of polydisperse packings: The effect of size span and shape of particle size distribution. Phys Rev E 96(2): 022902 (2017)

[40] Utter B, Behringer R P. Transients in sheared granular matter. Europ Phys J E 14: 373-380 (2004)

[41] DeGiuli E, McElwaine J N, Wyart M. Phase diagram for inertial granular flows. Phys Rev E 94(1): 012904 (2016)

Ph.D. candidate in mechanical design and theory at Hefei University of Technology, China. Her research interests include tribology and rheology of granular matter.

China. His current position is a professor and the director of the Institute of Tribology at Hefei University of Technology. His research areas cover tribology of granular matter, active control of interface friction, and powder metallurgy. 